\title{
Neutrophils activated by BJcuL, a C-type lectin isolated from Bothrops jararacussu venom, decrease the invasion potential of neuroblastoma SK-N-SH cells in vitro
}

\author{
Jessica Ohana Lemes Carneiro-Goetten', Bruna Santos Rodrigues², Rodrigo Amauri Nogoceke², \\ Thatyanne Gradowski do Nascimento ${ }^{1}$, Andrea Novais Moreno-Amaral', Patricia Maria Stuelp-Campelo², \\ Selene Elifio-Esposito2* (D) \\ 1Programa de Pós-graduação em Ciências da Saúde, Pontifícia Universidade Católica do Paraná (PUCPR), Curitiba, PR, Brazil. \\ ${ }^{2}$ Escola de Ciências da Vida, Pontifícia Universidade Católica do Paraná (PUCPR), Curitiba, PR, Brazil.
}

\section{Keywords:}

Snake venom

Neuroblastoma

SK-N-SH cells

C-type lectin

Polymorphonuclear leukocyte

Neoplasm invasion

\begin{abstract}
Background: Neuroblastoma is a pediatric tumor with a mortality rate of $40 \%$ in the most aggressive cases. Tumor microenvironment components as immune cells contribute to the tumor progression; thereby, the modulation of immune cells to a pro-inflammatory and antitumoral profile could potentialize the immunotherapy, a suggested approach for high-risk patients. Preview studies showed the antitumoral potential of BJcuL, a C- type lectin isolated from Bothrops jararacussu venom. It was able to induce immunomodulatory responses, promoting the rolling and adhesion of leukocytes and the activation of neutrophils.

Methods: SK-N-SH cells were incubated with conditioned media (CM) obtained during the treatment of neutrophils with BJcuL and FMLP, a bacteria-derived peptide highly effective for activating neutrophil functions. Then we evaluated the effect of the same stimulation on the co-cultivation of neutrophils and SK-N-SH cells. Tumor cells were tested for viability, migration, and invasion potential.

Results: In the viability assay, only neutrophils treated with BJcuL ( $24 \mathrm{~h}$ ) and cultivated with SK-N-SH were cytotoxic. Migration of tumor cells decreased when incubated directly $(\mathrm{p}<0.001)$ or indirectly $(\mathrm{p}<0.005)$ with untreated neutrophils. When invasion potential was evaluated, neutrophils incubated with $\mathrm{BJ}$ cuL reduced the total number of colonies of SK-N-SH cells following co-cultivation for $24 \mathrm{~h}(\mathrm{p}<0.005)$. Treatment with $\mathrm{CM}$ resulted in decreased anchorage-free survival following $24 \mathrm{~h}$ of treatment $(\mathrm{p}<0.001)$. Conclusion: Data demonstrated that SK-N-SH cells maintain their migratory potential in the face of neutrophil modulation by BJcuL, but their invasive capacity was significantly reduced.
\end{abstract}

\footnotetext{
* Correspondence: seleneesposito@gmail.com https://doi.org/10.1590/1678-9199-JVATITD-2019-0073 Received: 18 October 2019; Accepted: 01 April 2020; Published online: 11 May, 2020.
} 


\section{Background}

$\mathrm{BJcuL}$ is a lectin isolated from Bothrops jararacussu snake venom. It is a typical representative of the C-type animal lectin superfamily, having the carbohydrate recognition domain (CRD), and exhibiting specificity for $\beta$-D-galactosides [1]. Previous studies have shown that BJcuL has immunomodulatory effects. It can increase the adhesion and rolling of leukocytes in the endothelium of pre-capillary vessels [2], cause edema and increased vascular permeability when injected into mouse hind paw [3]. We have shown that BJcuL modulates macrophage differentiation towards a Th1 profile in vitro, with induction of phagocytosis, production of ROS [4], and secretion of proinflammatory cytokines, including TNF- $\alpha$, IL-6, IL-8, and GM-CSF $[4,5]$. BJcuL is also capable of modulating neutrophils to a pro-inflammatory phenotype, inducing the production of anion superoxide and increased phagocytic function [6].

In the tumor microenvironment (TME) cancer cells interact with extracellular matrix (ECM) proteins and non-tumor cells like fibroblasts, mesenchymal cells, and mature immune cells [7-9] that establish the overall characteristics of the tumor [10]. For example, cross talk between tumor cells and neutrophils can be a determinant in tumor progression. The first direct evidence of the cytotoxic effects of neutrophils on tumor cells was reported in 1972 [11], and since then many studies have contributed to this knowledge, as reviewed by Souto et al. [12]. Current data support a dual role for neutrophils in cancer biology, in which cytotoxic neutrophils, called N1, contribute to tumor rejection or increase immunological memory, thus combating tumor progression, and, on the other side of this spectrum, $\mathrm{N} 2$ neutrophils may enable tumor development, invasion, and metastasis [13].

Neuroblastoma (NB) is an extracranial tumor that may present during fetal development or early after birth, derived from the neural crest neuroepithelial cells [14]. It is characterized by heterogeneity and a broad spectrum of clinical behaviors ranging from spontaneous regression without any medical intervention to treatment resistant tumors with metastatic spread and poor patient survival [15-17]. Although there have been advances in the study, diagnosis, and treatment of NB, most patients with advanced disease do not enter remission even after treatment with multimodal therapies [18], which may include immunotherapy in the post-consolidation phase [19-22].

In this study, we investigate the potential of BJcuL to induce an $\mathrm{N} 1$ or antitumoral phenotype in neutrophils by analyzing the migration and invasion capabilities of NB cells following treatment and, thus, highlight the potential of using animal toxins and neutrophil modulation and showcase their use as effective weapons against chemotherapy-resistant solid tumors.

\section{Methods}

\section{Materials}

RPMI 1640 medium, fetal bovine serum (FBS), penicillin/ streptomycin and trypsin were purchased from GIBCO (USA).
Histopaque $^{\varpi} 1077$ and 1119, fMLP (N-formyl-L-methionyl-Lleucyl-L-phenylalanine), DCFH-DA (2',7'-Dichlorofluorescin diacetate) and methylene blue were all from Sigma-Aldrich (USA).

\section{Cells}

Human neuroblastoma cells SK-N-SH $\left(\right.$ ATCC $^{\circledR}$ HTB- $^{\mathrm{Tm}}{ }^{\mathrm{mm}}$ ) were cultured in RPMI 1640 medium supplemented with $100 \mathrm{IU} / \mathrm{mL}$ penicillin and $0.1 \mathrm{mg} / \mathrm{mL}$ streptomycin. Heat inactivated fetal bovine serum (FBS) was added to a final $10 \%$ concentration for culture expansion steps, or $2 \%$ for the scratch wound healing assay. The cultures were maintained in a humid incubator at $37^{\circ} \mathrm{C}$, with $5 \% \mathrm{CO}_{2}$.

Polymorphonuclear neutrophils (PMN) were isolated from peripheral blood of healthy adults aged 20-40 years using a Histopaque $^{\circledast}$ density gradient as described by the manufacturer with minor modifications (Figure 1A). Peripheral blood was centrifuged at $1000 \mathrm{x} g$ for $20 \mathrm{~min}$, and plasma was then centrifuged at $2000 \mathrm{x} g$ for $10 \mathrm{~min}$ to remove platelets. Plasma was then returned to the collection tube and homogenized with the remaining blood cells. Platelet-poor blood $(6 \mathrm{~mL})$ was added to tubes containing Histopaque $1119+1077(6 \mathrm{~mL})$, and centrifuged at $700 \mathrm{x} g$ for $45 \mathrm{~min}$. The PMN layer was collected and centrifuged at $700 \mathrm{x} g$ for $5 \mathrm{~min}$ to allow for the removal of residual Histopaque ${ }^{\circledast}$, and then mixed back into red blood cells (RBC) and plasma for a second round of centrifugation aiming to a better recovery of $\mathrm{PMN}$ without contamination with $\mathrm{RBC}$ or peripheral blood mononuclear cells (PBMC).

Aliquots of the PMN populations were analyzed on an Accuri ${ }^{\mathrm{TM}}$ C6 cytometer (BD Biosciences) for size (FSC, forward scatter) and complexity (SSC, side scatter) characterization (Figure 1B). A differential blood cell count (ABX MICROS 60 automatic counter) was performed before PMN isolation to verify that the cells were not pre-activated, based in the number of PMN cells in the complete blood count (CBC). Any alteration in percentage of PMN can indicate infection and thus a possible pre-activation of those cells. Therefore, only the blood which presented differential count with normal percentages of PMN was used.

\section{Bothrops jararacussu lectin (BJcuL)}

$B$. jararacussu venom was supplied by the Butantan Institute, São Paulo, Brazil. BJcuL was purified by affinity chromatography using an agarose-D-galactose column and characterized as previously described by Elifio-Esposito et al. [2].

\section{Stimulation of SK-N-SH cells}

SK-N-SK cells $\left(1 \times 10^{6}\right.$ cells $\left./ \mathrm{mL}\right)$ were co-cultured with neutrophils $\left(2.5 \times 10^{5}\right.$ cells $\left./ \mathrm{mL}\right)$ on a 24 -well plate $(300 \mu \mathrm{L} /$ well $)$, for $24 \mathrm{~h}$ in RPMI with or without BJcuL $(2.5 \mu \mathrm{g} / \mathrm{mL})$ or fMLP $(10 \mu \mathrm{M})$.

To obtain the conditioned media (CM), neutrophils $(2.5$ $\mathrm{x} 10^{5}$ cells $\left./ \mathrm{mL}\right)$ cultured in a 6 -well plate $(1 \mathrm{~mL} /$ well $)$ were incubated with BJcuL $(2.5 \mu \mathrm{g} / \mathrm{mL})$, fMLP $(10 \mu \mathrm{g} / \mathrm{mL})$, or $2 \%$ 


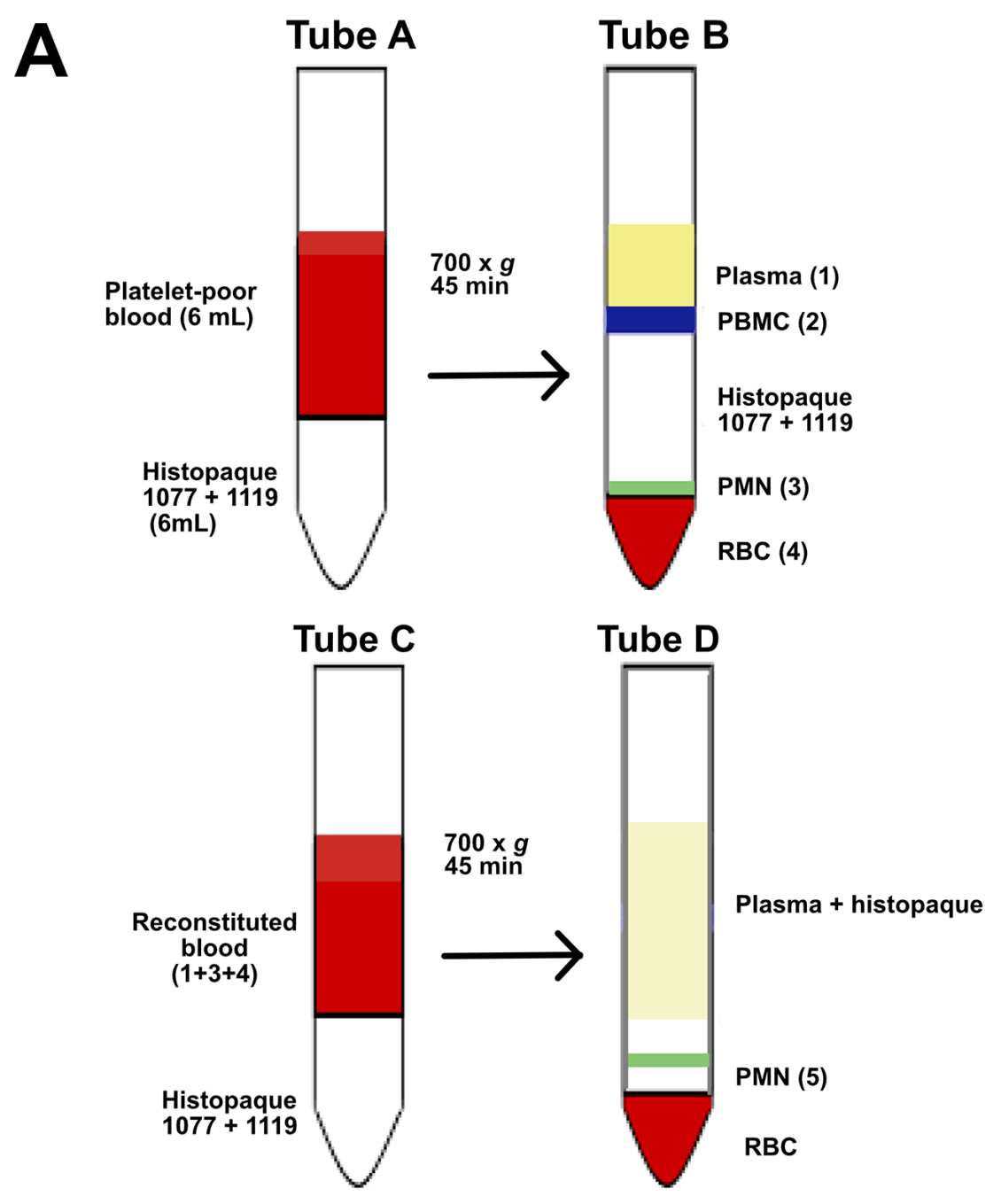

\section{B Layer 3}

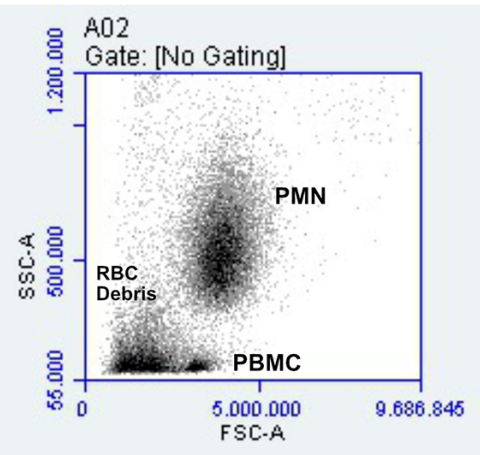

\section{Layer 5}

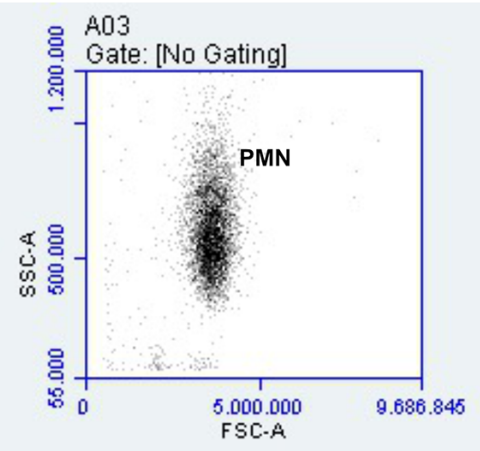

Figure 1. Isolation of neutrophils from human peripheral blood. Whole blood underwent a prior centrifugation step to isolate plasma and remove platelets. (A) Platelet-poor blood was used for the first fractionation step using Histopaque ${ }^{\circledR} 1077$ and 1119, resulting in four distinct layers: plasma, peripheral blood mononuclear cells (PBMC), polymorphonuclear neutrophils (PMN), and red blood cells (RBC). Plasma, PMN and RBC were reunited for a second isolation to allow us to obtain a PMN-enriched fraction. (B) Flow cytometry analysis of various fractions. Dot plot showing the mixture of PMN, RBC and PBMC in layer 3 , and the high concentration of PMN (layer 5). 
RPMI (untreated control) for $1 \mathrm{~h}$ or $24 \mathrm{~h}$. The supernatants were collected, centrifuged $(2000 \mathrm{x} g$ for $5 \mathrm{~min})$, filtered $(0.22 \mu \mathrm{m})$, and kept frozen at $-80^{\circ} \mathrm{C}$ until use. After the removal of the $\mathrm{CM}$, neutrophils from each treatment were stained with a DCFH-DA probe, according to the manufacturer's instructions and analyzed using an Accuri flow cytometer (BD Biosciences), to estimate the intracellular hydrogen peroxide level. For treatment of NB cells, CM was diluted 1:2 (v/v) with RPMI (2\% FBS) and added to wells containing the SK-N-SH tumor cells and incubated for $24 \mathrm{~h}$.

\section{Scratch Wound Healing (SWH)}

In vitro cell migration was evaluated using the scratch wound healing (SWH) method, according to the previously described protocol [23], with the following modifications. NB cells (3.5 $\mathrm{x} 10^{5}$ cells) were transferred to a 24 -well plate, and after $24 \mathrm{~h}$ the cell monolayer was mechanically scratched. Detached cells were removed by washing with RPMI. Cells were then treated for $24 \mathrm{~h}$, as described before. After treatment, cells were washed with RPMI and maintained in culture for another 24 h. Photomicrographs of the wounds were taken immediately after their formation $(0 \mathrm{~h})$ and at $48 \mathrm{~h}$ after (T48) using an EVOS $^{\circledast}$ XL microscope. The wound width at T0 and T48 was measured using the ImageJ software plugin (NIH, USA), and the migration distance was determined as the difference in width between $\mathrm{T} 0$ and $\mathrm{T} 48$.

\section{Cell viability assay}

SK-N-SH cell viability was determined directly on the plate using the SWH test, following the protocol described previously [24], with some modifications. Briefly, after completion of the SWH assay, medium was removed, and $200 \mu \mathrm{L}$ of methanol was added, and allowed to fix for $10 \mathrm{~min}$. Cells were then stained with $300 \mu \mathrm{L}$ of $0.05 \%$ methylene blue for $10 \mathrm{~min}$. Plates were washed by immersion and remained at room temperature for drying for $24 \mathrm{~h}$. About $300 \mu \mathrm{L}$ of $0.1 \mathrm{M} \mathrm{HCl}$ was added and plates were agitated for $10 \mathrm{~min}$. Following which $100 \mu \mathrm{L}$ from each well was transferred to a 96-well plate for absorbance reading on a microplate reader (ThermoPlate, TP Reader), at a wavelength of $630 \mathrm{~nm}$.

\section{Soft agar anchorage-free survival test}

The soft agar test was performed in 48-well plates as previously described [25]. The bottom layer was made up of $250 \mu \mathrm{L}$ of $2 \mathrm{X}$ RPMI medium containing $20 \%$ of FBS and $250 \mu \mathrm{L}$ of $1.2 \%$ agar in water. The upper layer consisted of $250 \mu \mathrm{L}$ of $2 \mathrm{X}$ RPMI medium (20\% FBS), $2 \times 10^{3}$ SK-N-SH cells, $125 \mu \mathrm{L}$ of $1.2 \%$ agar and $125 \mu \mathrm{L}$ of water. Every 72 h, $30 \mu \mathrm{L}$ of $1 \mathrm{X}$ RPMI medium (10\% FBS) was added to form the feeding layer. SK-N-SH cells were treated by the direct (BJcul or fMLP) or indirect method (CM) for $24 \mathrm{~h}$ prior to being added to the upper layer. Results were obtained by direct colony counting following 14 days of culture.

\section{Statistical Analysis}

The results for the quantitative tests are represented by the mean \pm standard deviation. Statistical analysis was performed by ANOVA, followed by multiple comparison testing using the Tukey's or Dunnett's test. Statistical significance was assigned for values where $\mathrm{p}<0.05$. Analyses were performed using GraphPad Prism Software, version 8.0 (La Jolla California, USA).

\section{Results}

\section{SK-N-SH cell migration is reduced in the presence of neutrophils and BJcuL reverses this effect}

The effects of BJcuL on NB cell migration were assessed by SWH assays following two distinct stimulation procedures. In the indirect method, NB cells were incubated for $24 \mathrm{~h}$ with conditioned media (CM) generated by culturing neutrophils in RPMI medium containing BJcuL or fMLP for $1 \mathrm{~h}$ (NE1h-CM) or $24 \mathrm{~h}$ (NE24h-CM). In the direct method, NB cells were cocultivated with neutrophils for $24 \mathrm{~h}$, in the presence of BJcuL or fMLP. We also verified the direct effects of BJcuL or fMLP on tumor cells, in the absence of neutrophils.

For the indirect assay, media obtained from non-stimulated neutrophils reduced the migration rate of SK-N-SH cells when compared to RPMI control, although a significant difference was found only for the $1 \mathrm{~h}$ group. Even so, for both $1 \mathrm{~h}$ or $24 \mathrm{~h}$ conditions, CM from neutrophils treated with BJcuL and fMLP improved cell migration compared to CM-RPMI, restoring the migration capacity, closer to RPMI control (Figure 2 and Additional file 1). Cell viability analysis was done to exclude the possibility that the behavior observed in the SWH assay was the result of cellular proliferation. The viability of the neuroblastoma cells was not affected in treatments with conditioned media.

Following the same pattern, the direct incubation with neutrophils (NE-RPMI) reduced SK-N-SH migration, which was restored to RPMI control values when BJcuL or fMLP were added to the system (Figure 3 ). When BJcuL was used as a direct stimulant for $1 \mathrm{~h}$ (data not shown) or $24 \mathrm{~h}$, it did not change the migration rate of SK-N-SH cells, when compared with untreated cells (RPMI). NB cell viability was not affected by BJcuL alone, but when it was added to the neutrophil coculture, we observed an almost $20 \%$ reduction in the number of viable cells (Figure 3).

\section{Neutrophils treated with BJcuL reduce the invasion of SK-N-SH cells}

The invasion capacity of tumor cells in vitro was determined using the soft agar method. The principle of this method is that malignantly transformed cells lose their anchorage dependence and can grow and form colonies within a semi-solid agar matrix. All tested conditions, i.e., with direct or indirect stimulation 


\section{NE1h-CM}
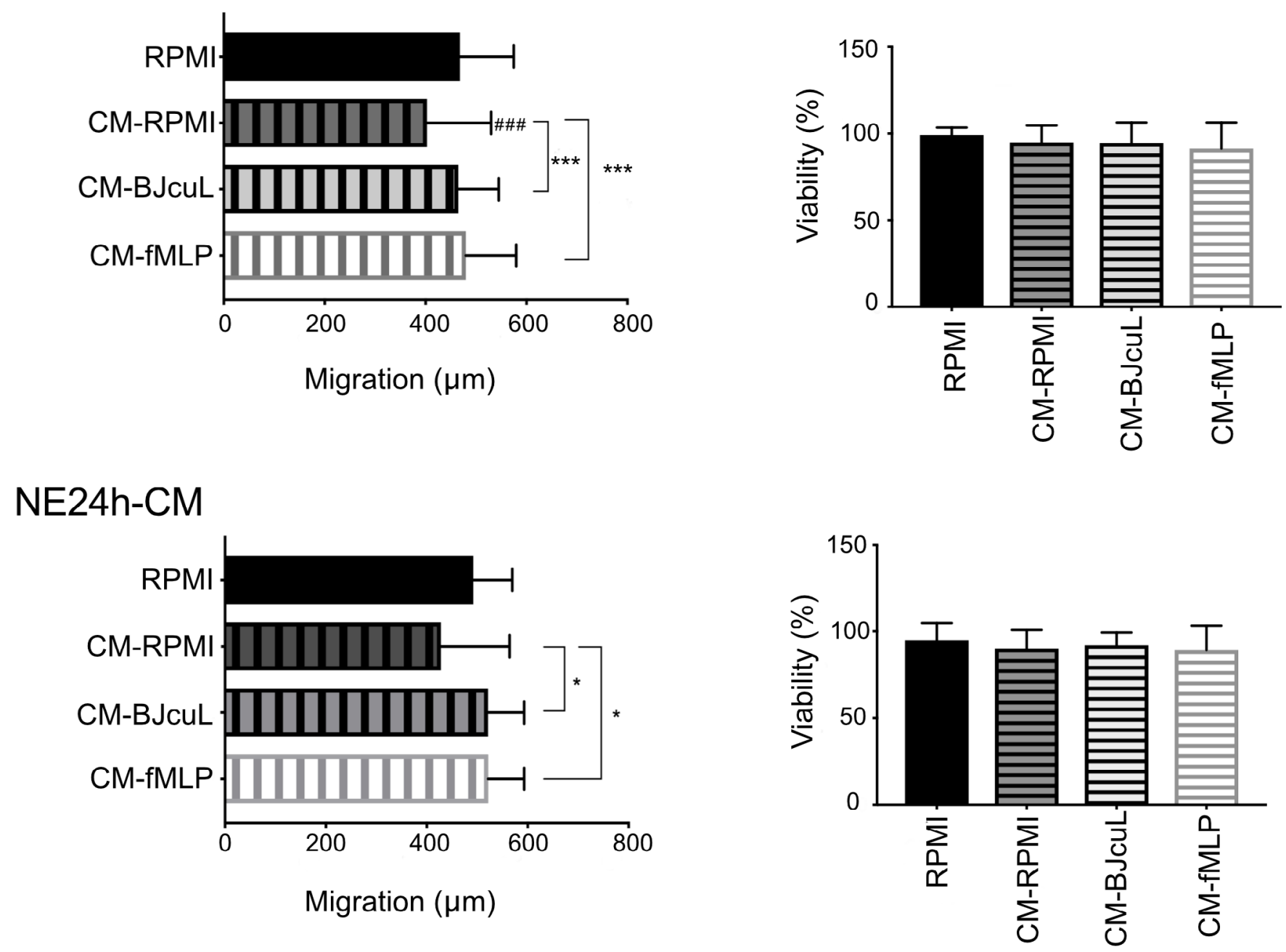

Figure 2. Analysis of migration and viability of SK-N-SH incubated with neutrophil conditioned media (CM). NB cells $\left(1 \times 10^{6}\right.$ cell/mL) were incubated for 24 $\mathrm{h}$ in a 24-well plate $(300 \mu \mathrm{L} /$ well) with 1:2 (v/v) neutrophil-conditioned media (CM). CMs were generated by culturing neutrophils in RPMI, $2.5 \mu \mathrm{g} / \mathrm{mL}$ BJcuL or $10 \mu \mathrm{M}$ fMLP for $1 \mathrm{~h}$ (NE1h-CM) or $24 \mathrm{~h}$ (NE24h-CM). Quantitative results are represented as means \pm standard deviation (SD) of the migration distance ( $\mu \mathrm{m}$ ), determined as the difference in wound width at T0 and T48 (T0-T48; $n=12)$. After the completion of the migration assay, the viability assay of the NB cells in each condition was established. Viability results are represented as mean $\pm S D(n=10)$ of viable cells normalized to the untreated control (RPMI). Data were obtained from at least three independent assays. Statistical analysis was performed by one-way ANOVA and Tukey's test with significance reported as *p $<0.05$; ${ }^{*} *_{p}<0.005$ and $* * * p<0.001$. \# indicates difference comparing with RPMI control.

of SK-N-SH cells, led to a decrease in the number of colonies when compared to the RPMI group. After $24 \mathrm{~h}$ of co-culture with neutrophils in RPMI alone (NE-RPMI) or in the presence of BJcuL or fMLP (NE-BJcuL; NE-fMLP), the invasion capacity of SK-N-SH was reduced by approximately $30 \%$, when compared to the untreated control (RPMI). Incubation of tumor cells with the conditioned media (CM-RPMI) also reduced the number of colonies, which was more pronounced for the CM from neutrophils treated with BJcuL (CM-BJcuL) and fMLP (CMfMLP), with a drop of $40 \%$ and $35 \%$ in the number of colonies, respectively, when compared with the RPMI control (Figure 4).

\section{BJcuL stimulates the production of hydrogen peroxide by neutrophils}

Neutrophils employed for the production of the CMs had their pro-inflammatory activity assessed by monitoring ROS production after incubation with BJcuL and fMLP for $1 \mathrm{~h}$ and $24 \mathrm{~h}$. It was added a $12 \mathrm{~h}$-stimulation condition to uncover the profile of ROS production along time, even if no CM was generated for this time point. BJcuL and fMLP stimulated cells showed a higher level of intracellular hydrogen peroxide when compared to the untreated control (Figure 5). For fMLP treated cells there was a significant increase after $1 \mathrm{~h}$ of stimulation, 

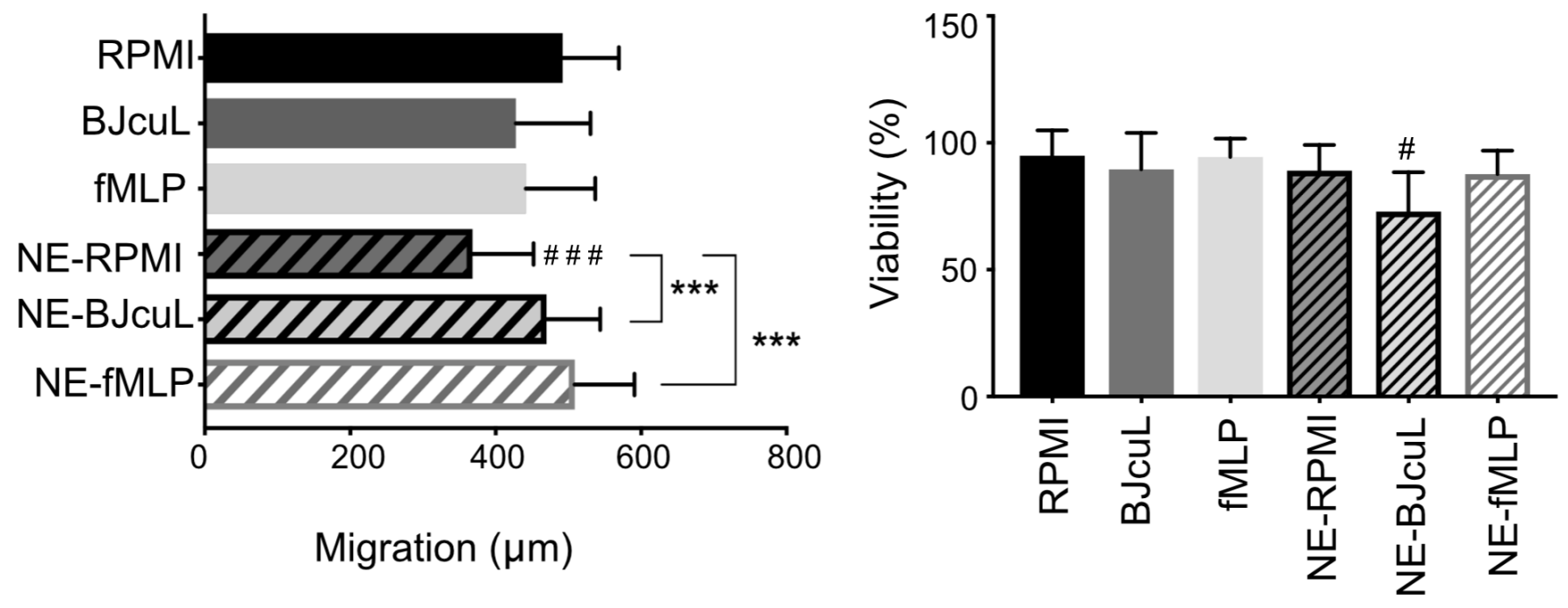

Figure 3. Analysis of migration and viability of SK-N-SH co-cultured with neutrophils. SK-N-SH cells $\left(1 \times 10^{6} \mathrm{cells} / \mathrm{mL}\right)$ were incubated with RPMI, $2.5 \mu g / \mathrm{mL}$ $\mathrm{BJcuL}$ or $10 \mu \mathrm{M}$ fMLP alone or in co-culture with human neutrophils (NE; $\left.2.5 \times 10^{5} \mathrm{cells} / \mathrm{mL}\right)$ for $24 \mathrm{~h}$ in a 24 -well plate $(300 \mu \mathrm{L} /$ well). Results represent mean \pm SD of the migration distance, determined as T0-T48 $(n=12)$. Viability results are represented as mean $\pm S D(n=10)$ of viable cells normalized to the untreated control (RPMI). Data were obtained from at least three independent assays. Statistical analysis was performed by one-way ANOVA and Tukey's test with significance shown as ${ }^{*} \mathrm{p}<0.05$ and ${ }^{* * *} \mathrm{p}<0.001$. \# indicates difference comparing with RPMI control.

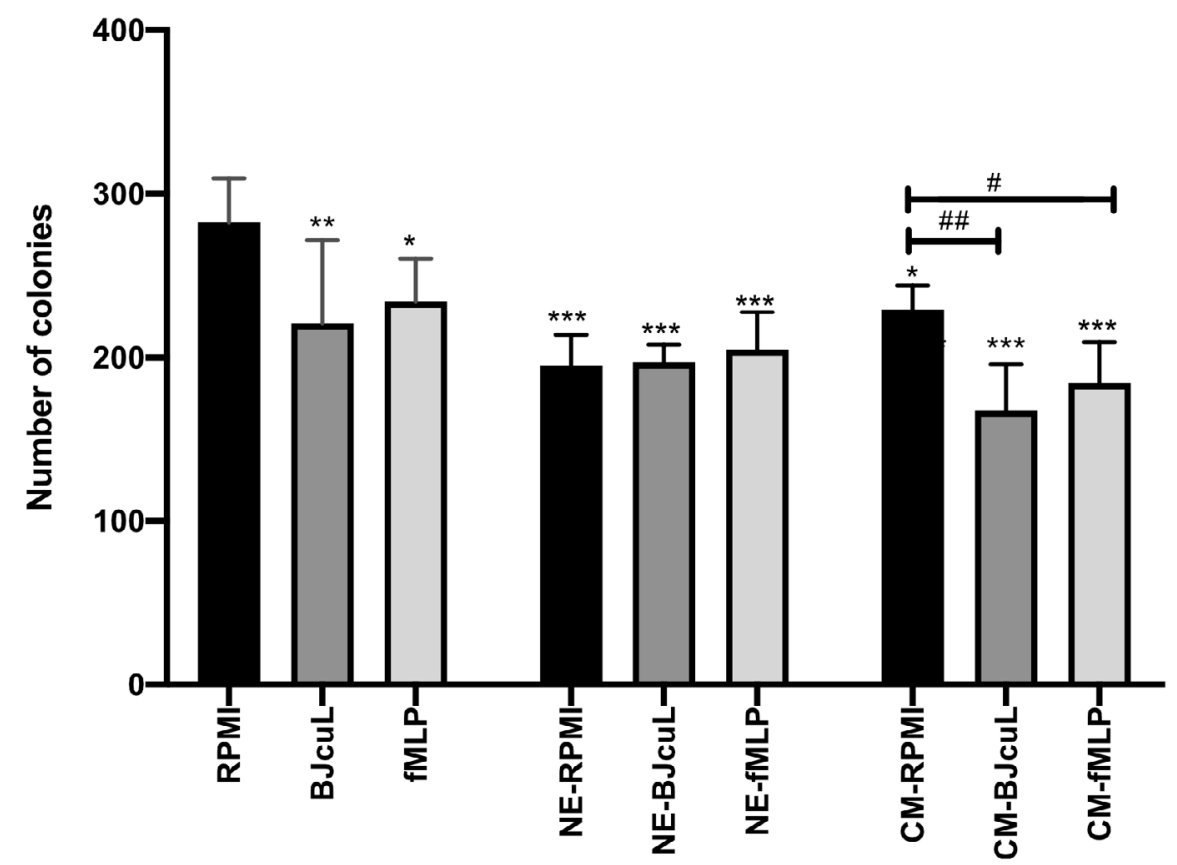

Figure 4. Anchorage-free survival assay of SK-N-SH cells after incubation with various stimuli. SK-N-SH cells $\left(2 \times 10^{3}\right.$ cells/well) were incubated for $24 \mathrm{~h}$ with RPMI, BJcuL $(2.5 \mu \mathrm{g} / \mathrm{mL})$ or fMLP $(10 \mu \mathrm{M})$, or conditioned neutrophil media (CM-RPMI, CM-BJcuL or CM-fMLP). SK-N-SH cells were also stimulated for $24 \mathrm{~h}$ with neutrophils in association with BJcuL, fMLP or $2 \%$ RPMI. After treatment SK-N-SH cells were dissociated, washed and plated on a mirror plate containing soft agar layers. Results are represented as mean $\pm S D(n=8)$ of the total number of colonies counted in each well. Data were collected from at least two independent assays. The statistical comparison among groups was performed by one-way ANOVA $(p<0.05)$. Dunnetts's test was used to compare all treatments with the RPMI group. Significance is represented as ${ }^{*} p<0.05 ;{ }^{*} p<0.005$ and ${ }^{* * *} \mathrm{p}<0.001$. \# represents the comparison of treatments with the respective control (CM-RPMI). 


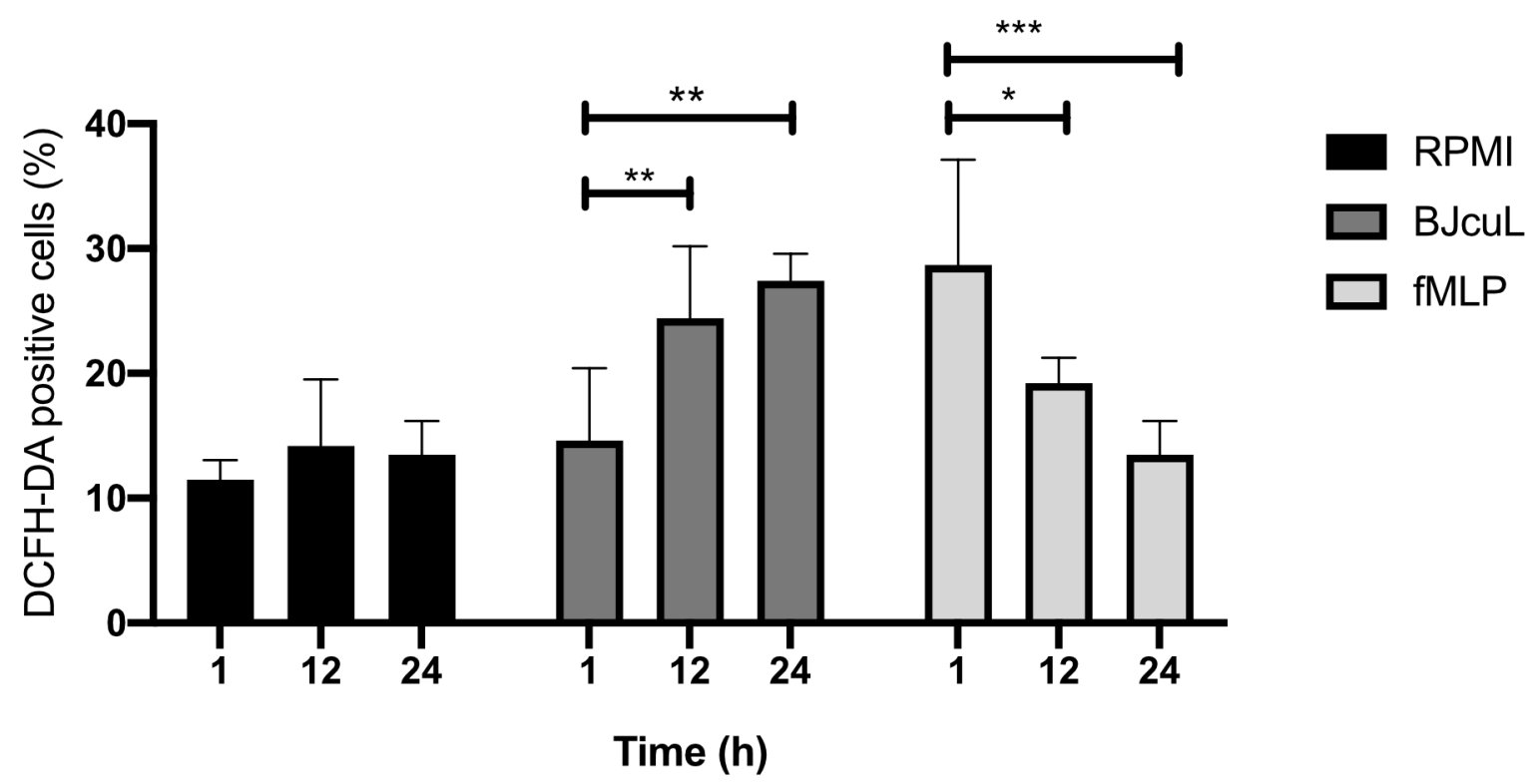

Figure 5. Determination of neutrophil intracellular hydrogen peroxide production. Neutrophils were treated with $\mathrm{BJcuL}(2.5 \mu \mathrm{g} / \mathrm{mL})$ or $\mathrm{fMLP}(10 \mu \mathrm{M})$ for 1 , 12 , and $24 \mathrm{~h}$ and stained with DCFH-DA for flow cytometric analysis. Results are represented as mean \pm SD. Statistical analysis was done using ANOVA and Dunnett's test for comparison of treated groups with untreated control (RPMI). Statistical significance is represented as $* p<0.05$, and $* * * p<0.001$.

which gradually reduced to the $24 \mathrm{~h}$ time point. BJcuL produced the opposite profile, with low intracellular ROS content after 1 $\mathrm{h}$ of stimulation, and a statistically significant increase in ROS production after $12 \mathrm{~h}$ and $24 \mathrm{~h}$.

\section{Discussion}

C-type lectins are among the main components exhibiting proinflammatory activity in viper snake venoms [1,26-28]. The pro-inflammatory potential of $B$. jararacussu venom lectin was reported previously [2-5, 29], which includes the induction of neutrophil polarization [6]. In this study, we demonstrated that the modulation of neutrophils by BJcuL reduced the viability and invasion capacity of human neuroblastoma cells in vitro. As neutrophil polarization is likely driven by TME [30, 31], neutrophils were tested in the presence and absence of tumor cells.

Exogenous chemotaxins, as fMLP, attract neutrophils to inflammatory sites to recognize and kill pathogens through a combination of cytotoxic mechanisms [32, 33]. Emerging evidence supports that neutrophils may also be cytotoxic to tumor cells [30,34]. Our results show that a significant reduction in SK-N-SH cell viability occurs when they are cultivated directly with neutrophils for $24 \mathrm{~h}$ in the presence of BJcuL, but not fMLP (Figures 2 and 3), which is consistent with the increasing levels of intracellular hydrogen peroxide found by BJcuL-treated neutrophils within $24 \mathrm{~h}$ (Figure 5). ROS-mediated cell killing was shown to be dependent on tumor cell expression of TRPM2, an $\mathrm{H} 2 \mathrm{O} 2$-dependent calcium channel, which upon activation results in a lethal influx of calcium ions into the cell $[35,36]$. The conditioned media generated by the cultivation of neutrophils did not affect NB cell viability, which may be due to the different in the concentration of the cytotoxic agents, as the CM was diluted for the incubation with NB cells.

As previously reported, BJcuL reduces the degree of late apoptosis in neutrophils, decreasing in $50 \%$ in the number of apoptotic cells after $24 \mathrm{~h}$ of incubation [6]. A recent study showed that mediators of delayed apoptotic cell death pathways of N1 cells can keep the system in the safe-guard immune N1 zone [37]. BJcuL could induce PMNs to exert a direct cytotoxic effect on tumor cells, and also favor the maintenance of an activated profile with effects on other aspects of tumor progression.

Metastasis remains the leading cause of death for patients with cancer [38]. It is a multistep process that involves the collective cell migration, which heavily relies on the cooperation of the cytoskeleton, the cellular surface adhesion proteins, and the extracellular matrix (ECM) components $[39,40]$. The secretion of cytokines by neutrophils, like components of the IL- 6 family and IL-8, for example, as well as growth factors [41-43], can promote ECM remodeling in the TME [94]. Furthermore, mature neutrophils possess granules containing a reservoir of enzymes, including myeloperoxidase (MPO), neutrophil elastase (NE), and also metalloproteinases (MMPs), capable of remodeling the ECM by stabilizing integrins [44]. Cell adhesion mediated mainly through the interaction of integrin receptors with their ECM ligands is a requirement for many cell types to proliferate and survive [45]. 
In this study, we found by SWH assay, a significant reduction in tumor cell migration when SK-N-SH cells were incubated directly with untreated neutrophils. These results were unexpected considering that circulating "normal" PMNs are described not to affect the adhesion and migration of tumor cells [46]. Regardless, the addition of either BJcuL or fMLP, both described to increase the pro-inflammatory potential of neutrophil, increased the migration capacity of NB cells. Neutrophil-tumor cell interaction was not the determining factor in this case, as the direct and indirect stimulation resulted in the same way. Furthermore, anchorage-free survival assays showed that incubation with untreated or stimulated neutrophils reduced the invasive power of neuroblastoma cells, which is even greater in the presence of BJcuL or fMLP.

The recognition of specific glycans by lectins represents a key event in a variety of biological phenomena involving cell-cell and cell-ECM component interactions [47, 48]. BJcuL, as Galatrox, another C-type lectin derived from B. atrox snake venom, can interact with glycans on neutrophils and macrophages surfaces, as well as with ECM proteins. They have been described also as stimulants of the neutrophils release of pro-inflammatory mediators, others than ROS, such as IL- 6 , and TNF- $\alpha[1,2,6$, 49]. These properties together could favor and stabilize cellular adhesion, facilitating migration but also reducing anchoragefree survival.

The complexity of the tumor microenvironment cannot be easily recapitulated in vitro. The master signals exchanged between various cell types within the tumor mass is critical, and evidence supports that cytokines control pro-tumor versus anti-tumor immune responses by polarizing neutrophil subpopulations to N1 or N2 phenotypes. However, even with the limitations, we reinforce in this study, that BJcuL affects the behavior of neutrophils in vitro and that such action can be a mechanism to be explored in order to better understand the processes involved in neutrophils anti-invasive action.

\section{Conclusion}

Our results showed that BJcuL-treated neutrophils could significantly affect the behavior of neuroblastoma cells in vitro. Viability was reduced when neutrophils were co-cultivated with the tumor cells, while the increment of cellular migration and the reduction of anchorage-free survival were also observed by the incubation of tumor cells with the neutrophil-conditioned media. Efforts must be made to identify the signaling molecules exposed on the cellular surface or secreted by neutrophils in order to explain the presented data.

\section{Abbreviations}

CBC: complete blood count; CM: conditioned media; CRD: carbohydrate recognition domain; ECM: extracellular matrix; FBS: fetal bovine serum; FSC: forward scatter; MMPs: metalloproteinases; MPO: myeloperoxidase; NB: neuroblastoma; NE: neutrophil elastase; PBMC: peripheral blood mononuclear cells; PMN: polymorphonuclear neutrophils; RBC: red blood cells; SSC: side scatter; SWH: scratch wound healing; TME: tumor microenvironment.

\section{Acknowledgments}

Authors would like to thank the Butantan Institute, São Paulo, Brazil for the supply of Bothrops jararacussu venom and Dr. Sandra M. W. Barreira, Federal University of Paraná, for her help in obtaining BJcuL.

\section{Availability of data and materials}

The dataset generated during and/or analyzed during the current study are available from the corresponding author on reasonable request.

\section{Funding}

This work was funded by the Araucaria Foundation for the Support of Scientific and Technological Development in the State of Paraná (FA-PBA/PUCPR 09/2016), Brazil, and the Coordination for the Improvement of Higher Education Personnel (CAPES-COFECUB 16/2015 \#880/2017, Finance Code 001).

\section{Competing interests}

The authors declare that they have no competing interests.

\section{Authors' contributions}

JOLCG, BSR, and RAN performed experiments and analysis. TGN, ANMA, and PMSC participated in the design and interpretation of the data. SEE conceived this research, designed experiments and wrote the paper. All authors read and approved the final manuscript.

\section{Ethics approval}

All the experimental procedures involving human participants were approved by the ethics committee of Pontifical Catholic University of Paraná (PUCPR) under the reference number 2.542.672.

\section{Consent for publication}

Not applicable.

\section{Supplementary material}

The following online material is available for this article:

Additional file 1. Representative photomicrographs of the wounds to the SK-N-SH cells monolayer taken at T0 and T48. 


\section{References}

1. Sartim MA, Sampaio SV. Snake venom galactoside-binding lectins: a structural and functional overview. J Venom Anim Toxins incl Trop Dis. 2015 Sep 24;21:35.

2. Elifio-Esposito SL, Hess PL, Moreno AN, Lopes-Ferreira M, Ricart CAO, Souza MV, et al. A C-type lectin from the venom of Bothrops jararacussu can adhere to extracellular matrix proteins and induce the rolling of leukocytes. J Venom Anim Toxins incl Trop Dis. 2007 Jan 30;13:782-99.

3. Panunto PC, da Silva MA, Linardi A, Buzin MP, Melo SE, Mello SM, et al. Biological activities of a lectin from Bothrops jararacussu snake venom. Toxicon. 2006 Jan;47(1):21-31.

4. Dias-Netipanyj MF, Boldrini-Leite LM, Trindade ES, Moreno-Amaral AN, Elifio-Esposito S. Bjcul, a snake venom lectin, modulates monocytederived macrophages to a pro-inflammatory profile in vitro. Toxicol In Vitro. 2016 Jun;33:118-24.

5. Pires WL, Kayano AM, de Castro OB, Paloschi MV, Lopes JA, Boeno $\mathrm{CN}$, et al. Lectin isolated from Bothrops jararacussu venom induces IL-10 release by TCD4. J Leukoc Biol. 2019 Sep;106(3):595-605.

6. Elifio-Esposito S, Tomazeli L, Schwartz C, Gimenez AP, Fugii GM, Fernandes LC, et al. Human neutrophil migration and activation by BJcuL, a galactose binding lectin purified from Bothrops jararacussu venom. BMC Immunol. 2011 Jan 25;12:10.

7. Borriello L, Seeger RC, Asgharzadeh S, DeClerck YA. More than the genes, the tumor microenvironment in neuroblastoma. Cancer Lett. 2016 Sep 28;380(1):304-14.

8. Hui L, Chen Y. Tumor microenvironment: Sanctuary of the devil. Cancer Lett. 2015 Nov 1;368(1):7-13.

9. Komohara Y, Takeya M. CAFs and TAMs: maestros of the tumour microenvironment. J Pathol. 2017 Feb;24;241(3):313-5.

10. Hashimoto $O$, Yoshida M, Koma $Y$, Yanai T, Hasegawa D, Kosaka $Y$, et al. Collaboration of cancer-associated fibroblasts and tumourassociated macrophages for neuroblastoma development. J Pathol. 2016 Oct;240(2):211-23.

11. Pickaver $A H$, Ratcliffe NA, Williams AE, Smith $H$. Cytotoxic effects of peritoneal neutrophils on a syngeneic rat tumour. Nat New Biol. 1972 Feb 9;235(58):186-7.

12. Souto JC, Vila L, Brú A. Polymorphonuclear neutrophils and cancer: intense and sustained neutrophilia as a treatment against solid tumors. Med Res Rev. 2011 May;31(3):311-63.

13. Piccard H, Muschel RJ, Opdenakker G. On the dual roles and polarized phenotypes of neutrophils in tumor development and progression. Crit Rev Oncol Hematol. 2012 Jun;82(3):296-309.

14. Johnsen JI, Dyberg C, Wickstrom M. Neuroblastoma - a neural crest derived embryonal malignancy. Front Mol Neurosci. 2019 Jan 29;12:9.

15. Diede SJ. Spontaneous regression of metastatic cancer: learning from neuroblastoma. Nat Rev Cancer. 2014 Feb;14(2):71-2.

16. Maris JM. Recent advances in neuroblastoma. N Engl J Med. 2010 Jun 10;362(23):2202-11.

17. Matthay KK, Maris JM, Schleiermacher G, Nakagawara A, Mackall CL, Diller L, et al. Neuroblastoma. Nat Rev Dis Primers. 2016 Nov 10;2:16078.

18. Lambert AW, Pattabiraman DR, Weinberg RA. Emerging biological principles of metastasis. Cell. 2017 Feb 9;168(4):670-91.

19. Ahmed M, Hu J, Cheung NK. Structure based refinement of a humanized monoclonal antibody that targets tumor antigen disialoganglioside GD2. Front Immunol. 2014 Aug 14;5:372.

20. Armideo E, Callahan C, Madonia L. Immunotherapy for highh-risk neuroblastoma: management of side effects and complications. J Adv Pract Oncol. 2017 Jan;8(1):44-55.

21. Cheung NK, Dyer MA. Neuroblastoma: developmental biology, cancer genomics and immunotherapy. Nat Rev Cancer. 2013 Jun;13(6):397-411.

22. Seeger RC. Immunology and immunotherapy of neuroblastoma. Semin Cancer Biol. 2011 Oct;21(4):229-37.

23. Lampugnani MG. Cell migration into a wounded area in vitro. Methods Mol Biol. 1999;96:177-82.
24. Oliver MH, Harrison NK, Bishop JE, Cole PJ, Laurent GJ. A rapid and convenient assay for counting cells cultured in microwell plates: application for assessment of growth factors. J Cell Sci. 1989 Mar;92(Pt 3):513-8.

25. Borowicz S, Van Scoyk M, Avasarala S, Karuppusamy Rathinam MK, Tauler J, Bikkavilli RK, et al. The soft agar colony formation assay. J Vis Exp. 2014 Oct 27(92):e51998.

26. Cezarette GN, Sartim MA, Sampaio SV. Inflammation and coagulation crosstalk induced by BJcuL, a galactose-binding lectin isolated from Bothrops jararacussu snake venom. Int J Biol Macromol. 2019 Feb 1;144:296-304.

27. Teixeira C, Fernandes CM, Leiguez E, Chudzinski-Tavassi AM. Inflammation induced by platelet-activation viperid snake venoms: perspectives on thromboinflammation. Front Immunol. 2019 Sep 4;10:2082.

28. Luna KPO, da Silva MB, Pereira VRA. Clinical and immunological aspects of envenomations by Bothrops snakes. J Venom Anim Toxins incl Trop Dis. 2011;17(2):11.

29. Pires WL, de Castro OB, Kayano AM, da Silva Setúbal S, Pontes AS, Nery NM, et al. Effect of BjcuL, a lectin isolated from Bothrops jararacussu, on human peripheral blood mononuclear cells. Toxicol In Vitro. 2017 Feb;41:30-41.

30. Fridlender ZG, Sun J, Kim S, Kapoor V, Cheng G, Ling L, et al. Polarization of tumor-associated neutrophil phenotype by TGF-beta: "N1" versus "N2" TAN. Cancer Cell. 2009 Sep 08;16(3):183-94.

31. Wu L, Saxena S, Awaji M, Singh RK. Tumor-Associated Neutrophils in Cancer: Going Pro. Cancers (Basel). 2019 Apr 19;11(4):pii: E564.

32. Selvatici R, Falzarano S, Mollica A, Spisani S. Signal transduction pathways triggered by selective formylpeptide analogues in human neutrophils. Eur J Pharmacol. 2006 Mar 03;534(1-3):1-11.

33. Amulic B, Cazalet C, Hayes GL, Metzler KD, Zychlinsky A. Neutrophil function: from mechanisms to disease. Annu Rev Immunol. 2012;30:459-89.

34. Rao X, Zhong J, Sun Q. The heterogenic properties of monocytes/ macrophages and neutrophils in inflammatory response in diabetes. Life Sci. 2014 Oct 29;116(2):59-66.

35. Yan J, Kloecker G, Fleming C, Bousamra M, Hansen R, Hu X, et al. Human polymorphonuclear neutrophils specifically recognize and kill cancerous cells. Oncoimmunology. 2014 Jul 3;3(7):e950163.

36. Giese MA, Hind LE, Huttenlocher A. Neutrophil plasticity in the tumor microenvironment. Blood. 2019 May 16;133(20):2159-67.

37. Kim Y, Lee D, Lee J, Lee S, Lawler S. Role of tumor-associated neutrophils in regulation of tumor growth in lung cancer development: A mathematical model. PLoS One. 2019 Jan 28;14(1):e0211041.

38. Liang W, Ferrara N. The Complex Role of Neutrophils in Tumor Angiogenesis and Metastasis. Cancer Immunol Res. 2016 Feb;4(2):83-91.

39. Spatarelu CP, Zhang H, Nguyen DT, Han XY, Liu RC, Guo QH, et al. Biomechanics of collective cell migration in cancer progression: experimental and computational methods. Acs Biomater Sci Eng. 2019 Aug;5(8):3766-87.

40. De Pascalis C, Perez-Gonzalez C, Seetharaman S, Boeda B, Vianay B, Burute $M$, et al. Intermediate filaments control collective migration by restricting traction forces and sustaining cell-cell contacts. J Cell Biol. 2018 Sep 3;217(9):3031-44.

41. Kerfoot SM, Raharjo E, Ho M, Kaur J, Serirom S, McCafferty DM, et al. Exclusive neutrophil recruitment with oncostatin $M$ in a human system. Am J Pathol. 2001 Oct;159(4):1531-9.

42. Chen MB, Hajal C, Benjamin DC, Yu C, Azizgolshani H, Hynes RO, et al. Inflamed neutrophils sequestered at entrapped tumor cells via chemotactic confinement promote tumor cell extravasation. Proc Natl Acad Sci U S A. 2018 Jul 3;115(27):7022-7.

43. Huh SJ, Liang S, Sharma A, Dong C, Robertson GP. Transiently entrapped circulating tumor cells interact with neutrophils to facilitate lung metastasis development. Cancer Res. 2010 Jul 15;70(14):6071-82.

44. Das A, Monteiro M, Barai A, Kumar S, Sen S. MMP proteolytic activity regulates cancer invasiveness by modulating integrins. Sci Rep. 2017 Oct 27;7(1):14219.

45. Fraley SI, Feng Y, Krishnamurthy R, Kim DH, Celedon A, Longmore GD, et al. A distinctive role for focal adhesion proteins in three-dimensional cell motility. Nat Cell Biol. 2010 Jun;12(6):598-604. 
46. Welch DR, Schissel DJ, Howrey RP, Aeed PA. Tumor-elicited polymorphonuclear cells, in contrast to "normal" circulating polymorphonuclear cells, stimulate invasive and metastatic potentials of rat mammary adenocarcinoma cells. Proc Natl Acad Sci U S A. 1989 Aug;86(15):5859-63.

47. Dan X, Liu W, Ng TB. Development and Applications of Lectins as Biological Tools in Biomedical Research. Med Res Rev. 2016 Mar;36(2):221-47.
48. Sharon $\mathrm{N}$, Lis $\mathrm{H}$. History of lectins: from hemagglutinins to biological recognition molecules. Glycobiology. 2004 Nov;14(11):53R-62R.

49. Sartim MA, Riul TB, Del Cistia-Andrade C, Stowell SR, Arthur CM, Sorgi $\mathrm{CA}$, et al. Galatrox is a C-type lectin in Bothrops atrox snake venom that selectively binds LacNAc-terminated glycans and can induce acute inflammation. Glycobiology. 2014 Nov;24(11):1010-21. 